\title{
Vehicle Routing Optimization for Surplus Food in Nonprofit Organizations
}

\author{
Ahmad Alhindi ${ }^{1}$, Abrar Alsaidi ${ }^{2}$, Waleed Alasmary ${ }^{3}$, Maazen Alsabaan ${ }^{4}$ \\ Computer Science Department, College of Computer and Information Systems \\ Umm Al-Qura University, Saudi Arabia, Makkah ${ }^{1,2}$ \\ Computer Engineering Department, College of Computer and Information Systems \\ Umm Al-Qura University, Saudi Arabia, Makkah ${ }^{3}$ \\ Computer Engineering Department, Computer Science and Information College \\ King Saud University, Saudi Arabia, Riyadh ${ }^{4}$
}

\begin{abstract}
Non-profit organizations mitigate the problem of food insecurity by collecting surplus food from donors and delivering it to underprivileged people. In this paper, we focus on a single non-profit organization located in Makkah city (Saudi Arabia), referred to as Ekram. The current surplus food pickup/delivery and operational routing model of Ekram organization have several apparent deficiencies. First, we model the surplus pickup/delivery and operational routing model as a vehicle routing problem (VRP). Then, we optimize the pickup/delivery of different types of food groups through the different available routes. Finally, we utilize the formulated VRP problem by minimizing the total route distances. Our proposed model ensures reduction of the total time and effort necessary to send the collecting vehicles to the donors of surplus food.
\end{abstract}

Keywords-Non-profit organization; vehicle routing problem; donor; surplus food; decision support

\section{INTRODUCTION}

Food waste is a globally critical issue and requires collective responsibility to be appropriately addressed. Fortunately, there exist several dedicated non-profit organizations to support food security by providing quality food surplus services [1]. The well-known charities that distribute food to underprivileged people in North America are Action Against Hunger, Feeding America and Food for the Poor. These organizations rely heavily on people donations. The donation-driven food delivery system is different from commercial supply chains because the commercial version rewards fast and cost-effective delivery as they generate extra profits [1]. While the food redistribution in non-profit organizations seeks to move the surplus food obtained through donations from the donors to the underprivileged people efficiently, the overarching objective is, therefore, nonprofit organization [2].

The St. Mary's Food Bank Distribution Centre adopted the heuristic concept that ensured efficiency in the track distribution system over the network. The organization relies on the services of about 330 agencies to enhance the efficient delivery of food products [3]. The VRP of the organization relies on the structured location of the agencies for better service delivery at minimal costs. The VRP models adopted by the food service organizations serve in the generation of minimum routes that serve most customers [4]. The problem of late collection of donated food staff from the donor and loading of tracks from the food banks severely affects food distribution since any delay accelerate the extent of wastage. Therefore, the presence of close monitoring and delivery of products significantly eliminate the probable long-term wastage in the service industry [5].

The food donations are different in terms of their quantity and frequency [1]. Hence, a comprehensive system is required to coordinate the collection and distribution of donations in an efficient mechanism. The mechanism has to take into consideration the following parameters: (1) avoid wasting the food, (2) reduce the delivery time, and (3) use the minimum number of drivers. In this paper, we model surplus food pickup/delivery operations of a non-profit organization in Makkah Province (Saudi Arabia) as an optimization problem, namely, a vehicle routing problem (VRP) that arise in many practical situations, e.g, pickup and delivery of goods to customers. The contributions of this proposed solution are as follows.

- We propose a novel routing model specifically designed for food surplus pick up/delivery systems to optimize route selection and time delivery.

- Improve the performance of the system by effective fleet management of transport.

- Improve vehicle utilization (one vehicle can pick up more than one package in a single route).

The rest of this paper is organized as follows. Section II provides a review of related works. Section III discusses the features of the Ekram organization. The proposed solution that will be used to solve the research problem is given in Section IV. Section V describes the proposed VRP model. Section VI describes the simulation study. Finally, the conclusion is given in Section VII.

\section{RELATED WORKS}

The implication of the Vehicle Routing Problem (VRP) uses an integrated optimization program to facilitate the logistics for the fleets of vehicles in managing the distribution systems. The programs facilitate the management of resources for efficient operations in complex networks. The efficiency with which the organizations manage the VRP define the sustainability of the enterprise in the non-profit entity platform [6]. 


\section{A. VRP in Food Delivery Organizations}

The Feeding Americans organization coordinates over 200 food banks and 60000 distributors [7]. It depends on a wellcoordinated network of the VRP. The organization uses the heuristic solution approach to create a balance between the routs and the duration spent to reach the community clients [7].

Another example is the OzHarvest which is a food bank in Australia [8]. The objective of this organization is collecting and redistributing foodstuffs to the welfare agencies supporting in the region based on the food recovery techniques to minimize wastes. The primary donations originate from restaurants and large food store outlets in the area. The advantages of this organization can be formulated in three points:

1) The focus on product specialization limits the extent of trash within the organization [8].

2) The organization implements successful training to the drivers to be able to check products at the collection point and enact reroute as deemed relevant based on the shelf life of the product donated.

3) The organization implements successful training to the drivers to be able to check products at the collection point and enact reroute as deemed relevant based on the shelf life of the product donated.

The use of VRP forecasting models facilitates efficiency in the organization through the implementation of overrun and underrun based on the peak threshold in the food supply platform [8].

Also, the Good Shepherd Food Bank is an example that should be mentioned in this article. This organization is located in Maine City [7]. It relies on food donations from the emergency food rescue team, the supermarkets, and the soup kitchen. In order to improve both the design and operations, the organization tends to collaborates with other food agencies. Such organizations encountered many constraints like the distance between the donation, consumer, and warehouse locations in addition to limited refrigeration facilities. Therefore, the focus on distribution network topology improves the level of VRP implementation in the organization [7]. The VRP design focuses on the population density of the consumer to facilitate re-routing of the delivery to accommodate efficiency in terms of management and operation of the agency outlets.

In [9], the researcher talks about the central depot of food supplies to other branches of Greater Bangkok and the losses experienced due to the perishability of food supplies. So, the central depot implements cooling trucks designed by routing and scheduling program in VRP. This minimized the loss of perishable goods such as vegetables. This goes a long way in saving food security in the city of Great Bangkok. It also creates the reliability of the central depot for the delivery of food supply.

A green logistic case study is provided in [10]. The researchers talk about Eroski, which is a Spanish supermarket chain, and highlights the challenges that the company suffers from late due to late deliveries. This study considers environmental pollution caused by the emission of several vehicles during deliveries of food products to the market [10].The delivery department at Eroski Company employed the VRP through the following methods:

1) It enabled drivers to deliver food supplies in time since VRP allows them to operate on different routes compared to the previous trucks where they could only access few routes.

2) It enabled the company to make fast deliveries.

3) It minimized the environmental pollution by taking the benefit of the fact that some vehicles can collect and deliver waste of food on various routes [10].

The last article that will be mentioned here is about Optimization of Vehicle Routing for Smart City. This article investigates the issue that supermarkets suffer from lack of availability of food products in the store due to lack of sufficient delivery in Casablanca [11]. The supermarkets in Casablanca addressed the issue of the unavailability of the onshelf product by integrating geographic information systems (GIS) with the VRP. The GIS was used to incorporate actual vehicle travel data and then choose the optimal structure to satisfy customers in order to minimize late deliveries and reduce vehicle congestion.

\section{EKRAM ORGANIZATION}

Ekram is a Saudi non-profit organization that officially declared by the Ministry of Labor and Social Development in 2007. This organization is located in the city of Makkah on the western side of Saudi Arabia. Ekram is a food rescue organization that plays a crucial role in alleviating hunger by preserving the food resources in the society by providing the complete process of picking up surplus food from donors up to delivering it the underprivileged people. The primary objective of Ekram is to create awareness on the importance of saving surplus food and not throwing it, through the provisioning process of it to the needy based on health requirements and distributed both meals and water for people in Ramadan and Hajj season. This organization's mission is saving the food by packaging the surplus food and delivering it to the underprivileged people in a hygienic condition. The organization's vision is to collect surplus, rapidly distributed using the best food safety standards (see http://www.ekram.org.sa).

Furthermore, the organization aims to manage the efficient communication and coordination of the collection of surplus food from donors and to distribute it to the underprivileged people. Ekram will also promote access to quality healthy food to the needy in society as a basic form of human wants. Considering that this organization's primary aim is to redistribute food resources in society, it is equipped with 40 vehicles of different capacities. The organization has also partnered with donors such as hotels as the sources of surplus food.

Ekram seeks to achieve the Saudi Vision 2030 through

- $\quad$ Reduce wastage of food by following the latest international standards and experiences.

- It is motivating people to volunteer.

One case that should be consider is when having more than a donor. In this case, several points should be taken in consideration, such as the fact that the food is going to be collected 
by different vehicles or depending on the availability of the automobiles (bear in mind here that there is little consideration of the route taken), the amount of the foods, the capacity of the vehicles (one vehicle delivered the food as a parts in several rounds from source to the destination), the proximity of multiple donors. Moreover, drivers will be required to decide depending on the kind and quality of surplus food collected from donors, on whether to distribute it to underprivileged people immediately or to deliver it to the center for sorting and packaging before entering the distribution phase. Efficient decision making by the drivers is based on several factors such as time, vehicle capabilities, and routing. Due to the lack of a well-defined vehicle planning system, the routing process in Ekram is often randomly.

The primary challenge facing Ekram is saving both time and efforts (in terms of sending vehicles to donors for collection of the surplus foods and how the requests can be managed).

\section{Proposed Solution for Ekram: Vehicle ROUTING PROBLEM}

The vehicle routing problem (VRP), also called route optimization, is described as a fleet of vehicles with different capacities, and a typical depot with several customers' demands to locate a set of routes that will offer minimum cost and serve all requests [12].

In the VRP, all the itineraries commence and terminate at the depot and are designed in such a way that each request is served only once by a single-vehicle [13]. The VRP is increasing the transport efficiency of a fleet of vehicles by finding the shortest route for each vehicle and considering realtime updates as the statuses of items change.

Theoretically, the VRP is a combinatorial optimization problem that may apply the integer programming technique to solve. The method seeks to determine the optimal set of routes for a fleet of the vehicle such as in the case of Ekram where the vehicles are expected to collect the surplus food and deliver in a depot where it can be distributed to the underprivileged people. The primary aim of the conventional vehicle routing techniques is to minimize the cost of a route. In the VRP employs several vehicles and set of routes; thus, the VRP is constrained by two vital elements, which are

1) Time constraints: every location in the route must visit within a particular period.

2) Capacity constraint: considering that the vehicles are required to collect items from each location, but a maximum capacity limits them.

The VRP will work on minimizing the length of the longest route for all vehicles. This assists in attaining the aim of VRP by completing all the deliveries within the shortest time possible.

\section{PRoposed VRP MOdEL}

The VRP aims to reduce both the time and effort involved in sending the vehicle to the donor for the collection of surplus food and the management of this problem. The VRP will be used to determine the low cost shortest path to service the charity demands.
In the case of Ekram is define on graph $\mathcal{G}=(\mathcal{N}, \mathcal{A})$, where $\mathcal{N}$ is the sets of nodes (destinations) and the $\operatorname{depot} \mathcal{N}=$ $\left\{n_{i}, \cdots, n_{m}\right\}$, at different locations. Every pair of locations are denoted by $(i, j), \mathcal{A}$ set of arcs such that $\mathcal{A}=\{(i, j)$ : $i, j \in \mathcal{N}, i \neq j\}, \mathcal{C}$ represents the set of donor associated with Ekram organization, $\mathcal{V}=\left\{v_{i}, \cdots, v_{m}\right\}$ is the sets of vehicles, $y_{i k}, y_{i j k}$ and $z_{i j k}$ are the binary variables, $x_{i j k}$ is the continuous variable, $L$ is the vehicle capacity, $i$ and $j$ are nodes, $k$ is the vehicles that have visited a node, and $d_{i}$ is the demand at the node.

We define binary decision variables $z_{i j k}$ equal to 1 if and only if in optimal solution vehicle $k$ visits customer $j$ after customer $i$, and $y_{i k}$ equal to 1 if and only if vertex $i$ is served by vehicle $k$. The problem is formulated as follows:

$$
\min _{z_{i j k}} \sum_{i \in \mathcal{A}} \sum_{j \in \mathcal{A}} c_{i j} z_{i j k} \quad k \in \mathcal{V}
$$

subject to the following terms:

$$
\begin{gathered}
\sum_{k \in \mathcal{V}} y_{i k} \geq 1 \quad i \in \mathcal{C} \quad \text { (Assignment) } \\
\sum_{(i, j) \in \mathcal{A}} z_{i j}=y_{i j k} \quad i \in \mathcal{N}, k \in \mathcal{V} \quad \text { (LeaveNode) } \\
\sum_{(i, j) \in \mathcal{A}} z_{i j}=y_{i j k} \quad i \in \mathcal{N}, k \in \mathcal{V} \quad \text { (EntryNode) } \\
\sum_{(i, j) \in \mathcal{A}} x_{i j k}-\sum_{(i, j) \in \mathcal{A}} x_{i j}=d_{i} y_{i k} \quad i \in \mathcal{C}, k \in \mathcal{V} \quad \text { (FlowBalance) }
\end{gathered}
$$

Determining the vehicle capacity.

$$
\begin{gathered}
x_{i j k}<L z_{(i j k)} \quad(i, j) \in \mathcal{A}, k \in \mathcal{V} \quad \text { (VehicleCapacity) } \\
y_{1 k}=1 \quad i \in \mathcal{C}, k \in \mathcal{V} \quad \text { (Depot) }
\end{gathered}
$$

The objective function aims to minimize the total traveled distance. Constraint (2) ensuring that each customer is served by at least one vehicle. The constraints (3) and (4) describing the arc within which the vehicle will leave or visit node $i$. The leave and entry node enforce the following condition: if node $i$ is visited by vehicle $k$ then vehicle $k$ will use one arc entering the node and then another arc that leaves node $i$. Additionally, if node $i$ is not visited by vehicle $k$, then no arc entering or leaving node $i$ should be used by vehicle $k$. Moreover, the constraint (5) describes the flow conservation at the nodes for each vehicle and constraint (6) ensure that no vehicle can be overloaded, the quantity of the product in each vehicle must always be less or equal to the vehicle capacity $L$. Finally, constraint (7) show when the vehicle is at the depot.

\section{Simulation StUdy}

\section{A. Simulation Setup}

We performed a simulation study of an organization similar to Ekram, and we studied the organization operating for a whole month. All the simulation experiments have been carried 
out on MacBook Pro (Intel Core i5 1.4GHz CPU and 8GB RAM). The programming language is Java.

First, we are assuming that there are 20 stores and 30 drivers or vehicles. Moreover, each store and driver has a unique ID number. Second, vehicles and drivers are uniformly distributed over an area of $(10 \mathrm{~km} \mathrm{x} 10 \mathrm{~km})$.

In our simulation, in every working hour, the organization takes the order from the store, which includes the store ID and the amount of surplus food. Then, the program selects the vehicle in order to send it to the store. Next, the program computes the distance between the selected vehicle and the store in order to divide the distance by average vehicle speed and computes the time for each vehicle. Finally, the program calculates the amount of food received by the end of each day.

Here, we assume that there are 20 orders for the next hour so that drivers will be assigned randomly to these orders. Fig. 2 shows the output that will be achieved.

After that we assume the same number of orders on the first case but with applying the VRP (involves the choice of short-distance routes), before assigning the driver to the order. Fig. 3 shows the output.

In this study, we will measure performance based on two of the performance metrics

1) Travel time: the time spent by the vehicle to reach its destination.

2) Travel distance: the route distance from the vehicle location to the destination.

We assume that each pixel is equal to two meters and the average speed of vehicle is $(80 \mathrm{~km} / \mathrm{h})$. So, the travel time will be calculated based on the following formula:

$$
\text { Travel time }=\text { distance } \div \text { speed }
$$

Moreover, the travel distance will be calculated based on the following formula:

$$
\text { Travel distance }=\text { speed } \times \text { time }
$$

\section{B. Simulation Results and Discussion}

In this section, we describe the results obtained by our simulation. The performance measures used to evaluate the results are the total distance driven (travel distance) and total travel time.

Fig. 1 shows a graph/map of the stores and drivers locations. The black point represents the stores while the green points represent the drivers Moreover, Fig. 2 shows the drawbacks of the random selection, which can be summarized in two points. The first one is that some drivers travel more than others and the second one is that some drivers assigned to stores far to them.

In the simulation study above, when applying the VRP on it, the program assigned a driver based on its location relative to the store, which will lead to using the shortest path, as shown in Fig. 3.

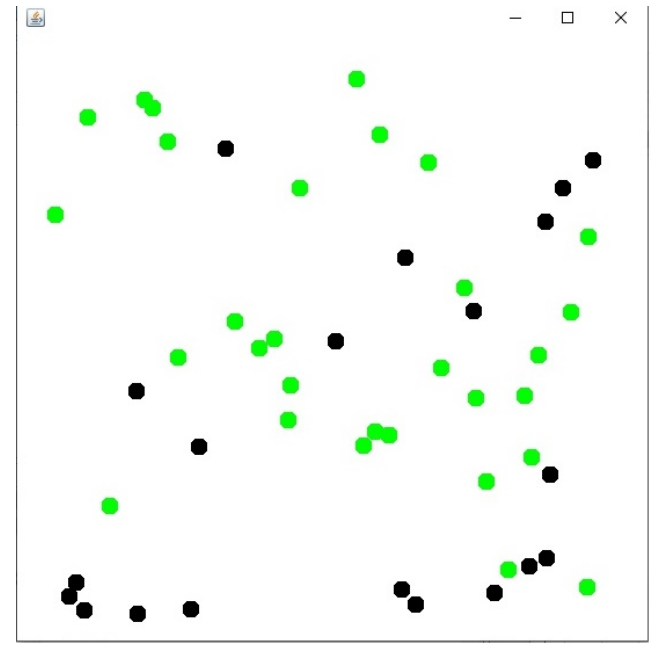

Fig. 1. An illustration of the location of the pick up/delivery points and the driver points on the hypothetical map. A one hour simulation scenario. The black point represents the stores while the green points represent the drivers.

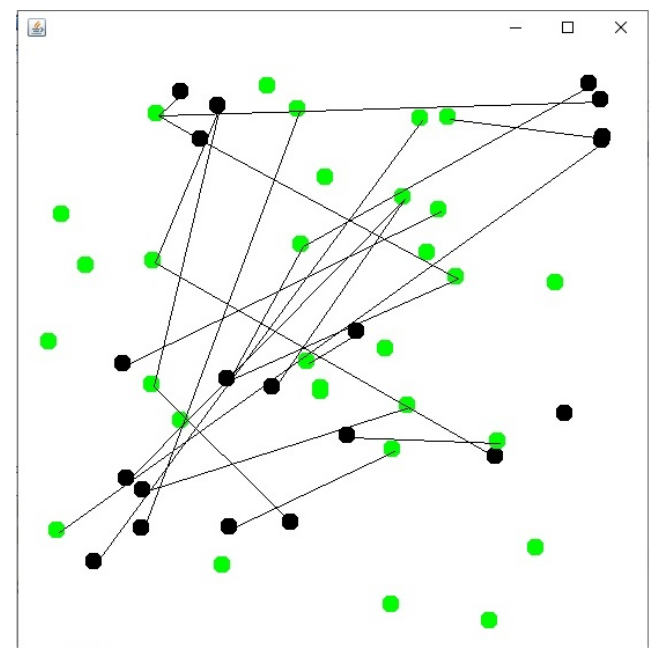

Fig. 2. An illustration of the simulation study to demonstrate the random mapping (i.e., creating a random graph/ assignment of drivers to pickup/delivery locations randomly). A one hour simulation scenario.

After implementing the simulation study for a complete month, the proposed VRP model (PRO) shows a smaller total for both travel time and distance when comparing to the travel time and distance obtained in the random model (RAND). Table I shows the total of travel time, travel distance, and number of the vehicle used at two models. At the bottom of the table, we add a row to show the utilization of the VRP model over the random model. The utilization is calculated in terms of percentage in terms of each of the above metrics as follows:

$$
\begin{aligned}
\text { Utilization }(\%) & =\left[\left(\mathrm{RAND}_{\text {metric value }}-\mathrm{PRO}_{\text {metric value }}\right)\right. \\
& \left.\div \mathrm{RAND}_{\text {metric value }}\right] \times 100
\end{aligned}
$$

Table I shows the improvement in travel time with 59.7\% and travel distance by $46.5 \%$ by using the VRP model comparing to the random model. This is because the VRP 
TABLE I. COMPARISON OF TRAVEL TIME (HOUR), TRAVEL DISTANCE (METER) AND NUMBER OF USED VEHICLES WITH RANDOM AND VRP MODELS. A ONE MONTH SIMULATION SCENARIO.

\begin{tabular}{|c|c|c|c|}
\hline & Travel time (hour) & Travel distance (meter) & Number of vehicles \\
\hline Random model & 467 & 64989494.0671 & 30 \\
\hline VRP model & 188 & 34746409.7178 & 17 \\
\hline Utilization \% & $59.7 \%$ & $46.5 \%$ & $43.3 \%$ \\
\hline
\end{tabular}

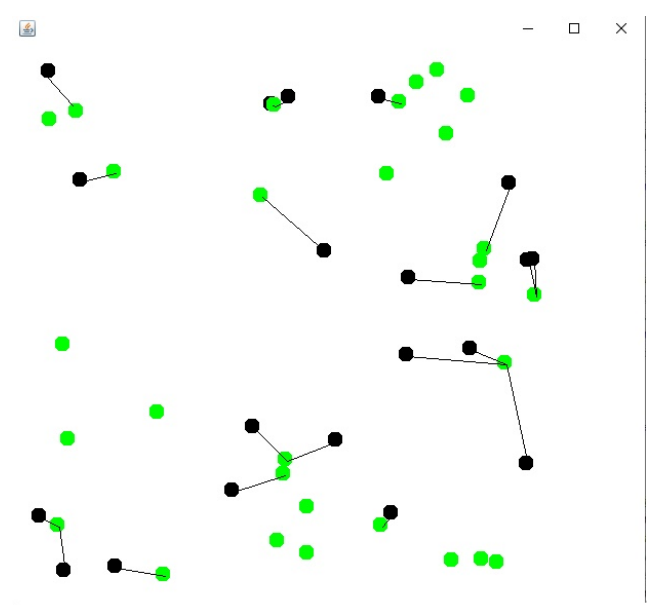

Fig. 3. An illustration of the simulation study to demonstrate the proposed VRP mapping with only 20 resulting links compared to Fig. 2. A one hour simulation scenario.

model searches for routes with the least distance before assign vehicles, which reduces the time is taken and enables one vehicle to service more requests. Thus, reduce the total number of vehicles used to satisfy the demands from 30 vehicles of the random model, it reduced to 17 vehicles of the VRP model. From that result, the reduction $43.3 \%$ in the VRP model.

For Ekram, VRP would be an excellent way to plan the collection and distribution of food effectively. However, the solution would have to be customized according to the needs and specific activities of the organization. For instance, a system for communicating the status of donors would need to implement, such that both the driver and the technical support team at the center can see whenever a new donation is available. Additionally, a database of common way points would also be necessary to increase the speed of the Route Optimizer. Based on the position of each of the 40 vehicles, the system can then suggest the most appropriate driver collect the new donation and determine whether it is more efficient to return it to the center or distribute it immediately.

Additionally, it would be necessary to incorporate information on the capacity of each vehicle, as well as any restrictions on the type of food it can carry. In such a way, the algorithm can compare the type and amount of food donated and determine the vehicle that should go and collect it. In the case of multiple donors, a vehicle can collect food at more than one point if it has enough capacity, and the most optimum route is selected for each automobile to avoid unnecessarily long trips or movement of near-empty vehicles.

When selecting the optimal path by Ekram, which is the shortest path with fewer efforts used to collect the food the following will be the outcomes:
- Enhance service quality by minimizing operational costs.

- Minimize the total distance of route (by determining the best path to reach the destination).

- Save both time and money used in the collection of food from the donor by using the optimal route.

- Improve the speed of movement of the vehicles between the depot and the food collection points.

- Increase the satisfaction of the needy people as well as the donors because both delivering and collecting waste of food will be done based on the scheduled plans.

- Minimize the number of required vehicles to meet the total demand.

By accounting for all ongoing and completed orders and employing advanced methods to analyze the route in real-time. VRP can tremendously increase transport efficiency. Besides that, it enhanced the quality and reliability of vehicle routing decisions by mitigating subjectivity in the decision-making process.

\section{CONCLUSION}

The need to collect and deliver multiple items in the shortest possible time while maintaining operational efficiency is a significant challenge for food delivery organizations. Ekram, which is a Saudi non-profit food saving organization, has a daily task of collecting and re-distributing surplus food in the city of Makkah. This daily routine of Ekram lacks a systematic decision support and efficient vehicle planning. One effective way to address this challenge is to utilize the vehicle routing problem (VRP) to better manage and determine the optimal set of routes a fleet of vehicles should take. In this paper, we demonstrate that the utilization of the VRP into the surplus food pickup/delivery model of Ekram organization results in an increased efficiency, reduced cost, and enhanced better management of the surplus food. Our simulation study demonstrated that our proposed model significantly outperforms the random pickup/delivery model in terms of the travel time and distance.

\section{REFERENCES}

[1] L. B. Davis, S. X. Jiang, S. D. Morgan, I. A. Nuamah, and J. R. Terry, "Analysis and prediction of food donation behavior for a domestic hunger relief organization," International Journal of Production Economics, vol. 182, pp. 26-37, 2016.

[2] D. J. Nair, T. H. Rashidi, and V. V. Dixit, "Estimating surplus food supply for food rescue and delivery operations," Socio-Economic Planning Sciences, vol. 57, pp. 73-83, 2017.

[3] X. Li, Capacitated Vehicle Routing Problem with Time Windows: A Case Study on Pickup of Dietary Products in Nonprofit Organization. Arizona State University, 2015. 
[4] N. Labadie, C. Prins, and C. Prodhon, "General presentation of vehicle routing problems," Metaheuristics for Vehicle Routing Problems, vol. 3, pp. 1-14, 2016.

[5] D. Goeke, R. Roberti, and M. Schneider, "Exact and heuristic solution of the consistent vehicle-routing problem," Transportation Science, vol. 53, no. 4, pp. 1023-1042, 2019.

[6] G. Erdoğan, F. McLeod, T. Cherrett, and T. Bektaş, "Matheuristics for solving a multi-attribute collection problem for a charity organisation," Journal of the Operational Research Society, vol. 66, no. 2, pp. 177190, 2015.

[7] L. B. Davis, I. Sengul, J. S. Ivy, L. G. Brock III, and L. Miles, "Scheduling food bank collections and deliveries to ensure food safety and improve access," Socio-Economic Planning Sciences, vol. 48, no. 3, pp. $175-188,2014$

[8] D. J. Nair, H. Grzybowska, D. Rey, and V. Dixit, "Food rescue and delivery: Heuristic algorithm for periodic unpaired pickup and delivery vehicle routing problem,' Transportation Research Record, vol. 2548, no. 1, pp. 81-89, 2016.

[9] K. Panapinun and P. Charnsethikul, "Vehicle routing and scheduling problems: a case study of food distribution in greater bangkok," Bangkok: Kasetsart University, 2005.

[10] S. Ubeda, F. J. Arcelus, and J. Faulin, "Green logistics at eroski: A case study," International Journal of Production Economics, vol. 131, no. 1, pp. 44-51, 2011.

[11] L. Safia, B. Jamal, A. Mustapha, M. Salma, and A. H. Sabry, "Optimization of vehicle routing for smart city: Real case study in casablanca," Smart Application and Data Analysis for Smart Cities (SADASC'18), 2018.

[12] H. Kurnia, E. G. Wahyuni, E. C. Pembrani, S. T. Gardini, and S. K. Aditya, "Vehicle routing problem using genetic algorithm with multi compartment on vegetable distribution," in IOP Conference Series: Materials Science and Engineering, vol. 325, no. 1. IOP Publishing, 2018, p. 012012.

[13] A. K. M. Masum, M. Shahjalal, F. Faruque, and I. Sarker, "Solving the vehicle routing problem using genetic algorithm," International Journal of Advanced Computer Science and Applications, vol. 2, no. 7, pp. 126131, 2011. 